\title{
Machine Learning Enabled Distributed Mobile Edge Computing Network
}

\author{
Junchao Ma \\ jcma@vt.edu \\ Bradley Department of Electrical and Computer \\ Engineering, Virginia Tech. \\ Blacksburg, Virginia \\ Key Lab of Information Coding and Transmission, \\ Southwest Jiaotong University \\ Chengdu, China \\ Pingzhi Fan \\ pzfan@swjtu.edu.cn \\ Key Lab of Information Coding and Transmission, \\ Southwest Jiaotong University \\ Chengdu, China
}

\author{
Hao-Hsuan Chang \\ haohsuan@vt.edu \\ Bradley Department of Electrical and Computer \\ Engineering, Virginia Tech. \\ Blacksburg, Virginia
}

\author{
Lingjia Liu \\ ljliu@vt.edu \\ Bradley Department of Electrical and Computer \\ Engineering, Virginia Tech. \\ Blacksburg, Virginia
}

\begin{abstract}
In this work, we propose to establish a mobile edge computing (MEC) network that considers computation, caching and communication jointly. Depending on the demanding categories, users in the network are partitioned into computation-driven and cachingdriven users, both of which need memory resource to improve their quality of experiences (QoEs). Thus, a memory resource allocation problem is aroused to maximize the performance of the whole network. Due to the fact that the users' characterization plays an important role to the resource allocation scheme and with the help of machine learning techniques, we propose to study and predict the users' patterns by distributed learning methods which take the heterogeneity of base station type and users' mobility, etc into consideration. The proposed machine learning based distributed MEC system can maximize the efficiency of the network by optimizing the resource allocation scheme and perfectly predicting users' pattern.
\end{abstract}

\section{CCS CONCEPTS}

- Networks $\rightarrow$ Network performance modeling; Network performance analysis.

\section{ACM Reference Format:}

Junchao Ma, Hao-Hsuan Chang, Pingzhi Fan, and Lingjia Liu. 2019. Machine Learning Enabled Distributed Mobile Edge Computing Network. In SEC '19: ACM/IEEE Symposium on Edge Computing, November 7-9, 2019, Arlington, VA, USA. ACM, New York, NY, USA, 2 pages. https://doi.org/10.1145/3318216. 3363454

Permission to make digital or hard copies of all or part of this work for personal or classroom use is granted without fee provided that copies are not made or distributed for profit or commercial advantage and that copies bear this notice and the full citation on the first page. Copyrights for components of this work owned by others than ACM must be honored. Abstracting with credit is permitted. To copy otherwise, or republish, to post on servers or to redistribute to lists, requires prior specific permission and/or a fee. Request permissions from permissions@acm.org.

SEC '19, November 7-9, 2019, Arlington, VA, USA

(C) 2019 Association for Computing Machinery.

ACM ISBN 978-1-4503-6733-2/19/11 . \$ \$15.00

https://doi.org/10.1145/3318216.3363454

\section{INTRODUCTION}

The synergy of Computing, Caching, and Communication (3C) has received much attention recently in $5 \mathrm{G}$ networks [4]. However, designing a network that jointly considers these three aspects is still an open problem [6]. The difficulty of the collaboration relies on the observation of user cases that relate to the three aspects simultaneously. In our work, we are the first to propose a machine learning based distributed mobile edge computing (MEC) network that jointly considers these three aspects. The users in the network are partitioned into two categories: caching-driven users with repeatedly requests of the same content (e.g. video on demand) [3] and computing-driven users with high computing power requirements (e.g. on-line gaming). The behaviors of user groups under the coverage of different base stations (BSs) are learned and predicted using advanced machine learning techniques $[1,5]$. Based on the prediction, we propose a resource allocation scheme to allocate the limited memory resource at BSs to caching resource and computation resource. Then the caching replacement and computation task offloading policy are developed to maximize the quality of experience (QoE) of caching-driven and computation-driven users, respectively. In addition, we propose an utility function to evaluate the QoE of users under the machine learning based MEC network.

\section{SYSTEM MODEL}

In our work, we consider a heterogeneous network consisting one macro BS (MBS) and a group of small BSs (SBSs). As shown in Fig. 1 , control information is exchanged between the MBS and SBSs or adjacent SBSs. Each edge BS (MBS or SBS) is equipped with a certain amount of memory which can be used to compute the offloaded tasks from users, or to cache contents users may retrieve. Depending on the BS type and some environment parameters (BS coverage, budgets, etc.), BSs are assumed to have heterogeneous memory capacities.

The users in the network are partitioned into two categories: caching-driven users and computation-driven users. Computationdriven users need to compute a series of tasks with stringent latency 


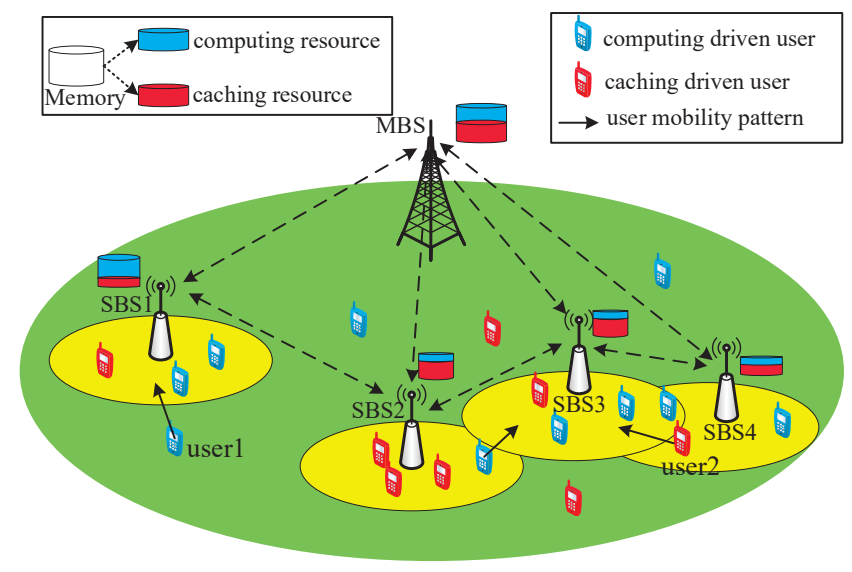

Figure 1: Applied Network Model.

requirements. To reduce the energy consumption at the users and the suffered latency which includes transmission delay and computation delay, the compute-intensive tasks are offloaded to edge BSs or other nearby users [2] and require large amounts of computation resource. On the other hand, caching-driven users need the forwarding of the edge BS when the requested streaming is from the cloud to the mobile user. To avoid redundant transmission by other requests of the same video content and to reduce the response latency, it is better that the edge BS caches some popular contents, which also requires large amount of storage resource [7]. Therefore, a problem arises: with limited memory resource equipped at the edge $B S$, how much of them should be allocated to computation resource and caching resource respectively? The problem can be addressed by carefully designing a resource allocation policy.

The prediction of the users' behavior patterns plays an important role in the network design. In contrast to existing works that assume knowing users' behavior patterns, in our work a machine learning based method is proposed to learn and predict users' behavior patterns. Our learning method is distributively ran in different BSs and different learning agents work collaboratively with one another through exchanging control information with adjacent BSs. To be specific, the heterogeneity of different BS capacities and coverage, users' mobility pattern, as well as the difference between local statistics and global statistics are considered in our learning algorithm. Given the prediction of users' behavior by the learning agents, the allocation of computation and caching resource can be done at each BS. Then the allocated computation and caching resource are used to serve different users' requirements to maximize their QoEs. We also propose an utility function to evaluate the QoE of task offloading performance and caching replacement performance. This function can be maximized by optimizing the task offloading decision and caching replacement policy together. In addition, the impact of communication is implicit in the resource allocation and thus $3 \mathrm{C}$ concept can be jointly considered in our proposed machine learning based distributed MEC network [4].

\section{ACKNOWLEDGMENTS}

The work of Junchao Ma and Pingzhi Fan is supported by the NSFC project under Grant No. 61731017, NSFC-NRF project under Grant No. 61661146003, and 111 project under Grant No. 111-2-14. The work of Hao-Hsuan Chang and Lingjia Liu is supported in part by National Science Foundation under Grant No. ECCS-1802710, ECCS-1811497, and CNS-1811720.

\section{REFERENCES}

[1] H. Chang, H. Song, Y. Yi, J. Zhang, H. He, and L. Liu. 2019. Distributive Dynamic Spectrum Access Through Deep Reinforcement Learning: A Reservoir ComputingBased Approach. IEEE Internet Things f. 6, 2 (April 2019), 1938-1948. https: //doi.org/10.1109/JIOT.2018.2872441

[2] H. Chen, L. Liu, H. S. Dhillon, and Y. Yi. 2019. QoS-Aware D2D Cellular Networks With Spatial Spectrum Sensing: A Stochastic Geometry View. IEEE Trans. Commun. 67, 5 (May 2019), 3651-3664. https://doi.org/10.1109/TCOMM.2018.2889246

[3] J. Ma, L. Liu, B. Shang, and P. Fan. 2019. Cache-aided Cooperative Device-to-Device (D2D) Networks: A Stochastic Geometry View. IEEE Trans. Commun. (2019), 1-1. https://doi.org/10.1109/TCOMM.2019.2931556

[4] A. Ndikumana, N. H. Tran, T. M. Ho, Z. Han, W. Saad, D. Niyato, and C. S. Hong. 2019. Joint Communication, Computation, Caching, and Control in Big Data Multi-access Edge Computing. IEEE Transactions on Mobile Computing (2019), 1-1. https://doi.org/10.1109/TMC.2019.2908403

[5] B. Shang and L. Liu. 2019. Machine Learning Meets Point Process: Spatial Spectrum Sensing in User-Centric Networks. IEEE Wireless Commun. Lett. (2019), 1-1. https://doi.org/10.1109/LWC.2019.2940442

[6] Y. Sun, M. Peng, Y. Zhou, Y. Huang, and S. Mao. 2019. Application of Machine Learning in Wireless Networks: Key Techniques and Open Issues. IEEE Communications Surveys Tutorials (2019), 1-1. https://doi.org/10.1109/COMST.2019.2924243

[7] Z. Zhang, C. Lung, M. St-Hilaire, and I. Lambadaris. 2019. An SDN-based Caching Decision Policy for Video Caching in Information-centric Networking. IEEE Transactions on Multimedia (2019), 1-1. https://doi.org/10.1109/TMM.2019.2935683 